\title{
Relação entre a Espessura Endometrial e os Niveis do B-HCG com a Resposta ao Tratamento da Gravidez Ectópica com Metotrexato
}

\author{
Relationship between Endometrial Thickness and $\beta$-HCG Levels in the \\ Response to Treatment of Ectopic Pregnancy with Methotrexate \\ Roberto da Costa Soares, Júlio Elito Júnior, Luiz Camano
}

\section{RESUM0}

\begin{abstract}
Objetivo: avaliar a relação entre os níveis séricos do hormônio gonadotrópico coriônico ( $\beta$ hCG) e a medida da espessura endometrial, em pacientes submetidas ao tratamento de gravidez ectópica com metotrexate.

Métodos: estudo clínico prospectivo, no qual foram avaliados os títulos do $\beta$-hCG e a maior medida da espessura endometrial no eixo longitudinal uterino por meio da ultra-sonografia transvaginal, em 38 pacientes com gravidez ectópica íntegra, com diâmetro $\leq 3,5 \mathrm{~cm}$, com estabilidade hemodinâmica e aumento dos niveis do $\beta$-hCG num intervalo de 24-48 horas. Todas receberam terapia com dose única de metotrexate (50 mg/ $\mathrm{m}^{2}$ intra muscular). Foram comparadas as médias dos valores do $\beta$-hCG e da espessura endometrial, das mulheres que evoluíram com sucesso do tratamento e com aquelas que apresentaram insucesso terapêutico. Posteriormente, foi avaliada a diferença entre as médias dos valores séricos do $\beta$-hCG segundo a espessura endometrial $(\leq 10,0 \mathrm{~mm}$ e $>10,0 \mathrm{~mm})$, independente da resposta terapêutica. Para análise estatística foi utilizado o teste t de Student.

Resultados: as médias dos valores iniciais do $\beta$-hCG das 28 mulheres que apresentaram resposta terapêutica medicamentosa foi de 1936,2 $\mathrm{mUI} / \mathrm{ml}$, significativamente menor do que a média das 10 mulheres sem resposta, 6831,3 mUI/ml (<0,04). Da mesma forma a média da espessura do endométrio foi significativamente menor nas mulheres com resposta $d$ que naquelas sem resposta, $6,4 \mathrm{~mm}$ e $11,7 \mathrm{~mm}$ respectivamente ( $p<0,01)$. A média dos valores do $\beta$-hCG das mulheres com espessura endometrial $\leq 10,0 \mathrm{~mm}$ foi $2008,7 \mathrm{mUI} / \mathrm{ml}$, significativamente menor que daquelas com endométrio $>10,0 \mathrm{~mm}$, cuja média foi de $6925,9 \mathrm{mUI} / \mathrm{ml}$.

Conclusões: a medida da espessura endometrial avaliada pela ultra-sonografia sofre influência dos níveis séricos do $\beta$-hCG, se mostrando valiosa como fator orientador adicional na indicação do tratamento medicamentoso com metotrexate na gravidez ectópica íntegra.
\end{abstract}

PALAVRAS-CHAVE: Gravidez ectópica. Endométrio: avaliação. Gonadotrofina coriônica.

\section{Introdução}

Apesar dos progressos no diagnóstico e tratamento da gravidez ectópica (GE), ela continua sendo a primeira causa de morte materna no primeiro

Departamento de Obstetrícia da Universidade Federal de São Paulo UNIFESP - EPM

Correspondência:

Roberto da Costa Soares

Av. Miguel Navarro y Canizares, 466, apto. 602

Edifício Vila Nobre - Pituba

41810-215 - Salvador - BA

Telefone: (71) 351-8242 $\quad$ (71) 9121-6521

FAX: (71) 351-8242

e-mail: mychelyroberto@uol.com.br trimestre da gestação e a segunda causa de mortalidade materna nos Estados Unidos da América, com incidência crescente nos últimos anos, atingindo uma proporção de 1,5-2,0\% de todas as gestações normais ${ }^{1-3}$. O desenvolvimento da ultrasonografia transvaginal (USTV) e a dosagem da fração beta do hormônio gonadotrópico coriônico ( $\beta$ hCG) auxiliam o diagnóstico mais precoce, permitindo a utilização de terapias conservadoras ${ }^{4}$.

A cirurgia conservadora (laparoscopia), o tratamento clínico com metotrexato (MTX) e a conduta expectante são condutas com resultados bastante positivos quando respeitados seus critérios de utilização ${ }^{5,6}$. 
Optando-se pelo tratamento medicamentoso com MTX, ele se mostra vantajoso por ser econômico ${ }^{7}$, podendo em casos selecionados ser realizado de forma ambulatorial e evitando os riscos da cirurgia e da anestesia. No caso de insucesso terapêutico, no entanto, colocamos a paciente diante de maiores riscos, pois neste período a GE pode evoluir para a rotura tubária, necessitando nesses casos de cirurgia de emergência, com maior morbidade e mortalidade, tornando obrigatória na maioria das vezes a realização da salpingectomia. Desta forma, nos casos de insucesso do tratamento com MTX, perde-se a oportunidade de realizar a cirurgia conservadora no momento do diagnóstico inicial.

O ponto principal para o sucesso terapêutico com MTX, e conseqüentemente a diminuição do número de falhas, é a rigorosa escolha das pacientes por meio de critérios de seleção. Alguns parâmetros orientadores já foram avaliados visando a mais adequada escolha das pacientes. Elito Junior et $a .^{8}$ descreveram o índice orientador ao uso do MTX empregando quatro variáveis: valor inicial do $\beta$-hCG, tamanho da massa anexial, aspecto da imagem à USTV e a dopplervelocimetria da massa. Gamzu et al. ${ }^{9}$ observaram que apenas as concentrações séricas do $\beta$-hCG apresentaram relação com o sucesso do tratamento. Nazac et al. ${ }^{10}$ notaram que a rota de administração do $\beta$-hCG e sua concentração sérica inicial são os mais importantes fatores associados com o sucesso terapêutico. Soares et al. ${ }^{6}$ demonstraram a validade da mensuração da espessura endometrial na indicação do tratamento com MTX, sendo observado que tal fato decorre de influências hormonais próprias do período gestacional inicial, que agem sobre o endométrio.

Resolvemos, neste estudo, avaliar se as concentrações séricas do $\beta$-hCG (influência hormonal) apresentam relação com este novo fator orientador do tratamento da GE íntegra com MTX, a espessura endometrial.

\section{Pacientes e Métodos}

Realizamos estudo prospectivo em 38 pacientes com diagnóstico de GE íntegra, submetidas ao tratamento medicamentoso com MTX no Departamento de Obstetrícia da Universidade Federal de São Paulo - Escola Paulista de Medicina (UNIFESP - EPM), entre abril de 1994 e julho de 2002. O estudo foi aprovado pelo Comitê de Ética em Pesquisa desta instituição. Carta de consentimento explicando o estudo foi assinada por todas as pacientes que participaram da pesquisa.
O diagnóstico de GE foi baseado na história clínica, exame físico, na dosagem e evolução dos títulos do $\beta$-hCG e sua associação com a USTV. Na gravidez tópica inicial, o título do $\beta$-hCG apresenta capacidade de duplicação em 1,4 a 3,5 dias ${ }^{11}$; já nas gestações inviáveis (GE e aborto), a elevação dos valores no período de 48 a 72 horas pode ser até de 66 ou 114\%, respectivamente, ocorrendo em $85 \%$ dos casos de gestações inviáveis ${ }^{12}$. Levando em consideração os valores do $\beta$-hCG e as imagens ultra-sonográficas, Kadar et al. ${ }^{13}$ descreveram: o diagnóstico de GE é extremamente provável se o útero não demonstrar saco gestacional intracavitário à luz da USTV, com valores do $\beta$ hCG superiores a 1.000 ou $2.000 \mathrm{mUI} / \mathrm{mL}$; nenhum diagnóstico pode ser definido na situação em que o $\beta$-hCG é menor que 1.000 ou 2.000 mUI/ $\mathrm{mL}$ com útero vazio; nestas situações, devemos realizar dosagens seriadas do $\beta$-hCG e nova USTV até elucidarmos o caso.

O $\beta$-hCG foi dosado pelo método imunoenzimométrico (Aia-Pack $\beta$-hCG, Tosoh Medic, Inc.), segundo o Terceiro Padrão Internacional ${ }^{7,14}$. A USTV foi realizada com o equipamento Synerg da General Eletric, sonda transvaginal de 7,5 MHz.

Confirmado o diagnóstico de GE pela associação entre os valores do $\beta$-hCG e a USTV, avaliamos a espessura endometrial da mesma forma como realizado por Spandorfer e Barnhart ${ }^{15}$, isto é, sua maior mensuração transversa no plano longitudinal uterino por meio da USTV. Posteriormente, avaliamos os ovários à procura do corpo lúteo e, por fim, fizemos a pesquisa de massas pélvicas, descrevendo suas características (hematossalpinge, anel tubário e embrião vivo) ${ }^{8}$ e tamanho.

Quando a massa anexial era menor ou igual a $3,5 \mathrm{~cm}$, as pacientes foram submetidas a duas dosagens do $\beta$-hCG quantitativo num intervalo de $24 / 48$ horas. Nos casos de massa maior que 3,5 $\mathrm{cm}$, foi indicado tratamento cirúrgico.

As pacientes com declínio dos valores do $\beta$ hCG em 24/48 horas foram submetidas a conduta expectante. Já os casos que apresentaram elevação ou manutenção dos títulos do $\beta$-hCG (38 casos) foram submetidos à terapia medicamentosa (MTX).

Assim, os critérios de inclusão empregados para o tratamento com MTX foram: estabilidade hemodinâmica, elevação ou manutenção dos valores do $\beta$-hCG no intervalo de 24 / 48 horas, diâmetro transverso da massa menor ou igual a 3,5 $\mathrm{cm}$, desejo de gravidez futura e consentimento para participar do estudo (documento aprovado pelo Comitê de Ética Médica em Pesquisa da UNIFESP).

Pacientes clinicamente instáveis, com sensibilidade prévia ao MTX, portadoras de discrasias 
sangüineas ou doenças hepáticas e renais foram excluídas do estudo.

As pacientes que preencheram os critérios de seleção receberam o MTX em dose única intramuscular (50 $\mathrm{mg} / \mathrm{m}^{2}$ de superfície corporal). No acompanhamento, os níveis séricos do $\beta$-hCG foram dosados no dia da utilização do MTX e no $4^{\circ} \mathrm{e}$ no $7^{\circ}$ dia após. A queda dos níveis séricos do $\beta$-hCG entre o $4^{\circ}$ e $7^{\circ}$ dia, associada à estabilidade clínica das pacientes, foi considerada indicador de boa evolução, e então estas pacientes receberam alta e passaram a realizar acompanhamento ambulatorial com dosagens semanais do $\beta$-hCG, até que os valores ficassem negativos. Quando as concentrações séricas do $\beta$-hCG se elevaram neste intervalo entre o $4^{\circ}$ e $7^{\circ}$ dia, ou a qualquer momento durante o acompanhamento ambulatorial semanal, foi indicada nova dose de MTX, ou tratamento cirúrgico de acordo com a aceitação da paciente.

$O$ critério de sucesso do estudo foi considerado o retorno dos valores do $\beta$-hCG a níveis negativos, sem a necessidade de realização da cirurgia.

Avaliamos então a média dos valores do $\beta$ hCG no momento da internação e a média da espessura endometrial, dos casos que evoluíram com sucesso do tratamento (MTX), comparando com os casos de insucesso terapêutico; para isto, empregamos o teste paramétrico $t$ de Student para duas amostras independentes. Fixou-se p<0,05 como o nível de significância estatística.

Posteriormente, dividimos as pacientes em dois grupos independentemente do resultado do tratamento, de acordo com a espessura endometrial. O primeiro grupo incluiu as pacientes com espessura endometrial menor ou igual a $10,0 \mathrm{~mm}$, ao passo que as pacientes com espessura maior que $10,0 \mathrm{~mm}$ formaram o segundo grupo. Avaliamos então a presença ou não de diferença estatística entre as médias das concentrações do $\beta$ hCG desses dois grupos, utilizando o teste paramétrico $t$ de Student. Também fixamos p<0,05 como o nível de significância estatística.

\section{Resultados}

O sucesso do tratamento medicamentoso com MTX ocorreu em 28 casos (73,7\%), já o insucesso ocorreu em 10 pacientes (26,3\%).

$\mathrm{Na}$ Tabela 1, apresentamos as médias dos valores do $\beta$-hCG no momento da internação, nos grupos sucesso $(1936,2 \mathrm{mUI} / \mathrm{mL})$ e insucesso da terapia com MTX (6831,3 mUI/mL). Os valores das medianas dos grupos sucesso e insucesso foram $1031,0 \mathrm{mUI} / \mathrm{mL}$ e $2300,0 \mathrm{mUI} / \mathrm{mL}$, respectiva- mente. Os valores máximos e mínimos para o grupo sucesso foram 14647,0 mUI/mL e 206,0 mUI/ $\mathrm{mL}$, ao passo que para o insucesso foram 37601,0 $\mathrm{mUI} / \mathrm{mL}$ e $310,0 \mathrm{mUI} / \mathrm{mL}$.

Tabela 1 - Média e desvio padrão dos valores do $\beta$-hCG e da espessura endometrial e 0 resultado do tratamento com methotrexate.

\begin{tabular}{lcc}
\hline Média & Sucesso & Insucesso \\
\hline$\beta-h C G ~(m U l / m l)$ & $1936,2(2900,9)^{\star}$ & $6831,3(11161,6)$ \\
Espessura $(\mathrm{mm})$ & $6,4(3,3)^{*}$ & $11,7(4,3)$
\end{tabular}

${ }^{*} \mathrm{~A}$ média dos níveis de $\beta$-hCG do grupo sucesso é significativamente menor que do grupo insucesso, $(p<0,04)$. Teste $t$ de Student para duas amostras independentes.

*A média da espessura endometrial do grupo sucesso é significativamente menor que a do grupo insucesso $(p<0,01)$.

A média da espessura endometrial entre os 28 casos de sucesso da terapia foi de $6,4 \mathrm{~mm}$ e das 10 pacientes que evoluíram com insucesso, 11,7 $\mathrm{mm}$. Os valores das medianas do grupo sucesso e insucesso foram $6,0 \mathrm{~mm}$ e $13,0 \mathrm{~mm}$, respectivamente. Os valores máximos e mínimos para o grupo sucesso foram, 3,0 $\mathrm{mm}$ e $18,0 \mathrm{~mm}$; enquanto para o insucesso foram 4,0 mm e 18,0 mm (p<0,04, teste $t$ de Student) (Tabela 1).

Para as médias da espessura endometrial, obteve-se $p<0,01$, mostrando haver diferença entre as médias dos valores do $\beta$-hCG e da espessura endometrial no momento da internação, entre as pacientes dos grupos sucesso e insucesso para o tratamento com MTX.

Na Tabela 2, apresentamos as médias dos valores do $\beta$-hCG para o grupo (26 casos) com espessura endometrial menor ou igual a $10,0 \mathrm{~mm}$ (2008,7 mUI/mL) e para o grupo (12 casos) com espessura maior que 10,0 mm $(6925,9 \mathrm{mUI} / \mathrm{mL})$. Ao avaliarmos a diferença entre as médias dos valores do $\beta$-hCG, entre o grupo com espessura menor ou igual a 10,0 mm e o grupo com espessura maior que $10,0 \mathrm{~mm}$ por meio do teste $t$ de Student, observamos que $\mathrm{p}<0,05$; portanto, existe diferença entre as médias dos valores séricos do $\beta$-hCG entre os dois grupos.

Tabela 2 - Média e desvio padrão dos valores do $\beta$-hCG nos diferentes valores da espessura endometrial.

Espessura endometrial

Menor ou igual 10,0 mm Maior que $10,0 \mathrm{~mm}$

$\beta$-hCG $(\mathrm{mUl} / \mathrm{mL} \pm \mathrm{DP}) \quad 2008,7 \pm 2520,9^{*} \quad 6925,9 \pm 11351,6$

${ }^{*}$ A média dos níveis de $\beta$-hCG do grupo com espessura menor ou igual a $10,0 \mathrm{~mm}$ é significativamente menor que do grupo com espessura maior que $10,0 \mathrm{~mm}(p<0,05)$, teste $t$ de Student para duas amostras independentes. 


\section{Discussão}

$\mathrm{Na}$ GE, o útero apresenta alterações semelhantes às que ocorrem numa gravidez tópica normal incipiente, como o amolecimento do colo e aumento do tamanho uterino. O tecido endometrial sob a ação hormonal é convertido em decídua, que apresenta características próprias como por exemplo a reação de Arias-Stella ${ }^{16}$. Tais modificações endometriais podem ser observadas de forma indireta pela USTV como endométrio espessado, caracterizado por tecido com reforço acústico posterior condicionado pelo edema endometrial ${ }^{17}$.

O sangramento vaginal em casos de GE é de origem uterina e está associado com a degeneração e descamação da decídua uterina. Nos casos de morte embrionária, ou seja, após a perda do suporte hormonal, a decídua sofre processo de degeneração e é eliminada pela descamação de pequenos fragmentos, originando o sangramento vaginal $^{18,19}$.

Soares et al. ${ }^{6}$ demonstraram a relação entre a espessura endometrial e o resultado do tratamento medicamentoso com MTX para casos de GE, baseando-se nestas alterações uterinas e endometriais que ocorrem durante o ciclo gravídico. Observaram que o endométrio fino (menor que 5,0 $\mathrm{mm}$ ), por estar submetido a menor influência hormonal, está mais associado ao sucesso da terapia, ao passo que o endométrio espesso (maior que $10,0 \mathrm{~mm}$ ) está associado ao fracasso do tratamento.

Os resultados de nosso estudo reafirmam estes achados ${ }^{6}$, demonstrando a diferença entre as médias da espessura endometrial do grupo sucesso $(6,4 \mathrm{~mm})$ versus insucesso $(11,7 \mathrm{~mm})$ do tratamento da GE com MTX. Como observado pela literatura, nossa pesquisa também demonstrou a influência das concentrações séricas do $\beta$-hCG sobre a terapia da GE com MTX, pois as concentrações elevadas do $\beta$-hCG estão associadas ao insucesso do tratamento, ao passo que niveis séricos baixos cursam em geral com sucesso terapêutico. Já em nossa última análise estatística, realmente confirmamos a influência hormonal das concentrações séricas do $\beta$-hCG sobre a espessura endometrial nos casos de GE, sendo notado que os casos que apresentaram endométrio menos espesso (menor ou igual 10,0 $\mathrm{mm}$ ) estavam associados a valores séricos mais baixos do $\beta$-hCG (média de 2008,7 mUI/mL), ao passo que valores mais elevados do $\beta$-hCG (média de 6925,9 $\mathrm{mUI} / \mathrm{mL}$ ) estavam associados a endométrio mais espesso (maior que 10,0 mm).

A espessura endometrial reflete, portanto, a ação hormonal do $\beta$-hCG e, como demonstrado, quanto maior o título do $\beta$-hCG no momento da internação, maior é a espessura endometrial e como conseqüência pior será o prognóstico para o tratamento da GE com MTX.

Concluímos que a espessura endometrial avaliada pela USTV pode funcionar como critério orientador adicional aos outros já existentes, do tratamento da GE com MTX (50 mg/m²), permitindo o aperfeiçoamento da seleção das pacientes, diminuindo os casos de insucesso da terapia.

\section{ABSTRACT}

Objective: to evaluate the correlation between the beta-human chorionic gonadotropin ( $\beta-h C G)$ serum levels and the measurement of the endometrial thickness, in patients under treatment of ectopic pregnancy with methotrexate.

Methods: a prospective study in which the levels of $\beta-h C G$ as well as the largest measurement of the endometrial thickness on the uterine longitudinal axis through transvaginal ultrasound were evaluated at 24-48 h intervals in thirty-eight patients with hemodynamic stability, ectopic pregnancy, diameter $\leq 3.5 \mathrm{~cm}$, and increased $\beta$ - $h C G$ levels. All the patients got methotrexate in a single-dose therapy $\left(50 \mathrm{mg} / \mathrm{m}^{2} \mathrm{im}\right)$. We compared the mean values of $\beta-h C G$ and endometrial thickness of cases that evolved successfully versus the poor responders using the Student t-test. Afterwards we analyzed the difference of the $\beta$ - $h C G$ mean serum values related to the endometrial thickness $(\leq 10.0 \mathrm{~mm}$ and $>10.0 \mathrm{~mm})$ independently of the response to treatment employing the Student $t$-test.

Results: the mean values of $\beta$ - $h C G$ and endometrial thickness in patients with successful treatment (28 cases) were 1936.2 $\mathrm{mIU} / \mathrm{ml}$ and $6.4 \mathrm{~mm}$, respectively, significanlty lower than the mean values for insuccessful cases: $6831.3 \mathrm{mIU} / \mathrm{ml}$ and $11.7 \mathrm{~mm}$, respectively $(p<0.05)$. The mean values of $\beta-h C G$ in women with endometrial thickness $\leq 10.0 \mathrm{~mm}$ were 2008.7 $\mathrm{mIU} / \mathrm{ml}$, significantly lower than the ones with endometrium $>10.0 \mathrm{~mm}$, whose mean values were $6925.9 \mathrm{mIU} / \mathrm{ml}(<0.05)$. Conclusions: the measurement of the endometrial thickness through ultrasound is under the $\beta-h C G$ serum values influence, and it showed to be a valuable additional factor to suggest medical treatment with methotrexate in the nondisrupted ectopic pregnancy.

KEYWORDS: Ectopic pregnancy. Endometrium. Chorionic gonadotropin.

\section{Referências}

1. Coste J, Job-Spira N, Aublet-Cuvelier B, Germain E, Bouyer J, Fernandez H. Stability of incidence rates of ectopic pregnancy. Results of a population- 
based register in France, 21 ème Congrès de L Association des Epidémiologistes de Langue Française, Bruxelles 3-5 Juin 1996. Arch Public Health 1996; 56:32.

2. Pisarska MD, Carson SA. Incidence and risk factors for ectopic pregnancy. Clin Obstet Gynecol 1999; 42:2-8.

3. Department of Health. Report on confidential enquiries into maternal deaths in the United Kingdom 1994-1996. London: HMSO; 1998.

4. Soares RC, Elito Junior J, Camano L. Avaliação ultra-sonográfica na gravidez ectópica: normas adotadas no Departamento de Obstetrícia da UNIFESP. Femina 2003; 31:147-51.

5. Buster JE, Pisarska MD. Medical management of ectopic pregnancy. Clin Obstet Gynecol 1999; 42:2330.

6. Soares RC, Elito Junior J, Hann KK, Camano L. Espessura endometrial como fator orientador do tratamento clínico da gravidez tubária íntegra. Rev Bras Ginecol Obstet 2002; 24:309-14.

7. Sowter MC, Farquhar CM, Gudex G. An economic evaluation of single dose systemic methotrexate and laparoscopic surgery for the treatment of unruptured ectopic pregnancy. BJOG 2001; 108:204-12.

8. Elito Junior J, Reichmann AP, Uchiyama MN, Camano L. Predictive score for the systemic treatment of unruptured ectopic pregnancy with a single dose of methotrexate. Int J Gynaecol Obstet 1999; 67:75-9.

9. Gamzu R, Almog B, Levin Y, et al. Efficacy of methotrexate treatment in extrauterine pregnancies defined by stable or increasing human chorionic gonadotropin concentrations. Fertil Steril 2002; 77:761-5.

10.Nazac A, Gervaise A, Bouyer J, de Tayrac R, CapellaAllouc S, Fernandez H. Predictors of success in methotrexate treatment of women with unruptured tubal pregnancies. Ultrasound Obstet Gynecol 2003; 21:181-5.
11.Fritz MA, Guo SM. Doubling time of human chorionic gonadotropin (hCG) in early normal pregnancy: relationship to hCG concentration and gestational age. Fertil Steril 1987; 47:584-9.

12.Graczykowski JW, Seifer DB. Diagnosis of acute and persistent ectopic pregnancy. Clin Obstet Gynecol 1999; 42:9-22.

13.Kadar N, DeVore G, Romero R. The discriminatory hCG zone: its use in the sonographic evaluation for ectopic pregnancy. Obstet Gynecol 1981; 58:15661.

14. Olofsson JI, Poroma IS, Ottander U, Kjellberg L, Damber MG. Clinical and pregnancy outcome following ectopic pregnancy; a prospective study comparing expectancy, surgery and systemic methotrexate treatment. Acta Obstet Gynecol Scand 2001; 80:744-9.

15.Spandorfer SD, Barnhart KT. Endometrial stripe thickness as a predictor of ectopic pregnancy. Fertil Steril 1996; 66:474-7.

16.Arias-Stella J. Atypical endometrial changes associated with the presence of chorionic tissue. AMA Arch Pathol 1954; 58:112-28.

17.Fleischer AC, Cartwright PS, Pennell RG, Sacks GA. Sonography of ectopic pregnancy with transabdominal and transvaginal scanning. In: Fleischer AC, Romero R, Buker DM, editors. The principles and practice of ultrasonography in obstetrics and gynecology. $4^{\text {th }}$ ed. New York: Prentice Hall; 1991. p. 3976.

18.Jones HO, Brewer JI. Arterial phenomena associated with uterine bleeding in tubal pregnancy. Am J Obstet Gynecol 1939; 38:839-61.

19.Cunningham FG, MacDonald PC, Gant NF, et al. Ectopic pregnancy. In: Williams Obstetrics. $20^{\text {th }}$ ed. East Norwalk: Appleton \& Lange; 1997. p. 607-34.

Recebido em: 10/11/2003 Aceito com modificações em: 10/5/2004

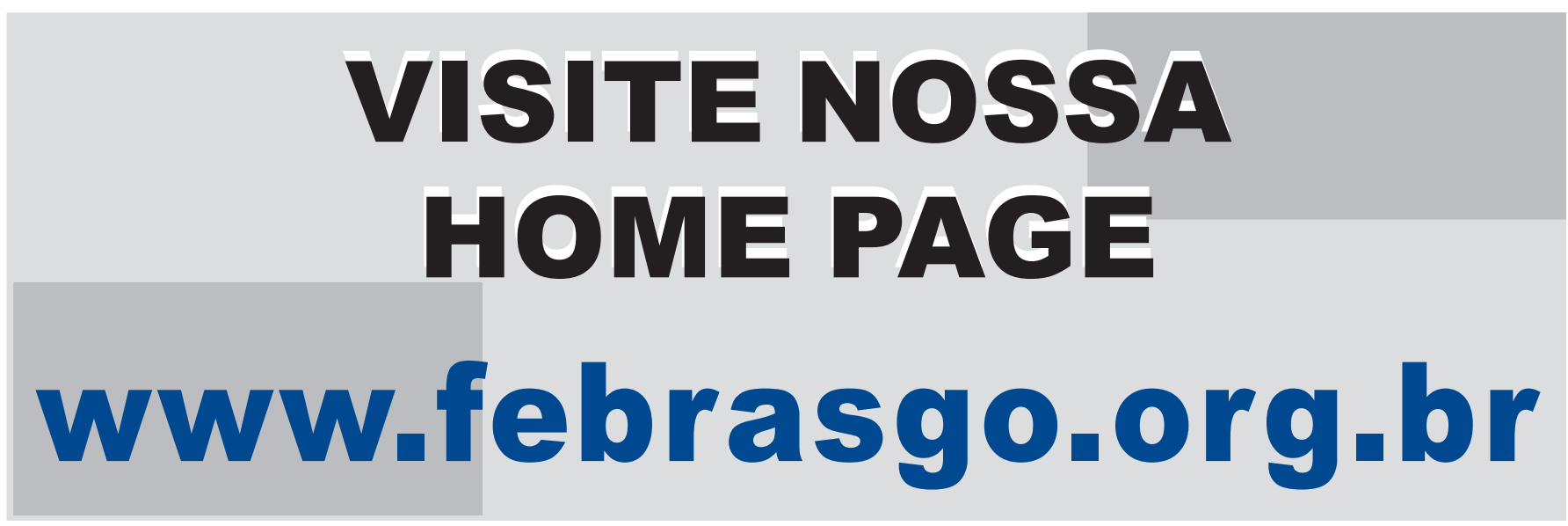

